Kybernetes

26,3

316

\section{Pansystems optimization, generalized principles of optimality, and fundamental equations of dynamic programming}

\author{
Beifang Chen
}

Hong Kong U niversity of Science and Technology, Kowloon, Hong Kong

\section{Introduction}

In the theory of dynamic programming (Bellman, 1957; Bellman and Dreyfus, 1962), the Bellman principle of optimality was not called into question until Rudd and Blum found a wrong solution for a chemical reaction system (Jackson, 1963; Rudd and Blum, 1962). A Ithough the Bellman principle of optimality is true for almost all practical problems, there are still some problems, both theoretical and practical, for which the principle is not valid (Denardo, 1967; Hu, 1982; Wu and Wu, 1984, 1986). Thus the recursive fundamental (functional) equation based on the Bellman principle of optimality cannot be unconditionally reliable. The importance of dynamic programming in practice lies in its technique of finding the recursive functional equation. It is therefore necessary to find conditions under which the recursive equation holds (Boltyanski, 1966; Wu, 1980). A variety of such conditions have been found since the 1960s.

The present paper was inspired by the questions asked by $\mathrm{Hu}(1982)$ and the work of Qin, Wu and others (Furukawa, 1980; Henig, 1983; Hu, 1982; Qin, 1981a, 1981b, 1983; Wu, 1980, 1984a, 1984b; Wu and Wu, 1984, 1986) on pansystems methodology. We consider the foundational problems of dynamic programming in view of whole-part relations and body-shadow relations of pansystems analysis (Wu, 1982, 1983, 1984a). W hile much of the analysis was completed many years ago (1984), the results are still new today. Our main objective is to clarify the logical relation between the Bellman principle of optimality and the recursive equation of dynamic programming. In the next section, we introduce the concept of optimum operators, which can be maximization, minimization, Pareto optimization, and symbolic optimization, etc. With the concept of optimum operators we establish the global-local principle and transformation principle of optimality. The two principles form a conservation principle of

The author thanks Professor X.-M. Wu for his helpful discussions.
Kybernetes, Vol. 26 No. 3, 1997 pp. 316-333 @ M CB University Press, 0368-492X 
optimality which is equivalent to the fundamental recursive equation of dynamic programming. In the third section, a generalized prototype of dynamic programming is set up, and a necessary and sufficient condition for the recursive equation of the model is obtained. The relation between the Bellman principle of optimality and the fundamental recursive equation is clearly stated in a precise set-theoretic language. In the fourth section, the discrete model is treated in detail by optimum operators. The advantage of optimum operators is that some problems that cannot be handled by classic dynamic programming could be solved by constructing certain appropriate optimum operators to satisfy the conservation principle of optimality. A $n$ example is given to demonstrate such a possibility.

\section{Generalized principles of optimality}

Definition 1. Let $X$ be a non-empty set, and let $P(X)$ be the power set of $X$. $A$ mapping $\theta: P(X) \rightarrow P(X)$ is called an optimum operator on $X$ if $\theta(A) \subset A$ and $\theta(B) \cap A \subset \theta(A)$ for any subsets $A, B$ of $X$ of such that $A \subset B$.

The optimum operator is a generalized concept of optimization. $\theta(A) \subset A$ can be interpreted as the collection $\theta(A)$ of "good" choices from $A$, so it is contained in $A$. Under the condition $A \subset B, \theta(B) \cap A \subset B \quad \theta(A)$ means that if an optimal element $x$ of $B$, namely, $x \in \theta(B)$, belongs to $A$, then $x$ should be an optimal element of $A$, namely, $x \in \theta(A)$. Maximization can be considered as a special optimum operator. For instance, the maximization on the set of real numbers induces an optimum operator $\max : P(\mathbf{R}) \rightarrow P(\mathbf{R})$ by $\max (A)=\max \{a \mid a \in A\}$, while searching the minimal element in a preordered set $(X, \leq)$ can be viewed as an optimum operator $\min : P(X) \rightarrow P(X)$ by $\min (A)=\{X \in A \mid$ there is no $a \in A$ such that $a<x$.

Global-local principle of optimality: Let $\theta$ be an optimum operator on a nonempty set X. If

$$
\theta\left(\bigcup_{\lambda \in \Lambda} W_{\lambda}\right)=\theta\left(\bigcup_{\lambda \in \Lambda} \theta\left(W_{\lambda}\right)\right)
$$

for any collection $W_{\lambda}(\lambda \in \Lambda)$ of subsets of $X$, then $\theta$ is said to satisfy the globallocal principle of optimality on $X$.

This principle, as an example of whole-part relations, is the abstraction of everyday common sense. Suppose there is a set $X$ of commodities for which we need to choose "good" ones $\theta(W)$ from a part $W$ of $X$, that is, $W \subset X$, if the size of $W$ is very large or the elements of $X$ are quite complicated, then it is not easy to determine the good ones. However, we may partition $W$ into some small parts $\mathrm{W}_{\lambda}(\lambda \in \Lambda)$, that is, $\mathrm{W}=\cup_{\lambda \in \Lambda} \mathrm{W}_{\lambda}$, so that each small part $\mathrm{W}_{\lambda}$ becomes easy to be chosen as the good ones $\theta\left(\mathrm{W}_{\lambda}\right)$. Then we may collect all those $\theta\left(\mathrm{W}_{\lambda}\right)(\lambda \in$ $\Lambda$ ) and select the good ones $\theta\left(\cup_{\lambda \in \Lambda} \theta\left(\mathrm{W}_{\lambda}\right)\right)$ from $\cup_{\lambda \in \Lambda} \theta\left(\mathrm{W}_{\lambda}\right)$, and these should be the good ones from $W$. 
Kybernetes 26,3

318
Proposition 1. Let $\theta$ be an optimum operator on a non-nempty set $X$. Let $W$ and $\mathrm{W}_{\lambda}(\lambda \in \Lambda)$ be subsets of $\mathrm{X}$ such that $\mathrm{W}=\cup_{\lambda \in \Delta} \mathrm{W}_{\lambda}$. Then

$$
\theta(\theta(A))=\theta(A), \quad \forall A \subset X, \text { i.e., } \theta^{2}=\theta,
$$

$$
\theta(W) \subset \theta\left(\bigcup_{\lambda \in \Lambda} \theta\left(W_{\lambda}\right)\right)
$$

Proof. Note that $\theta(\theta(A)) \subset \theta(A)$ follows from the first property of $\theta$. Since $\theta(A)$ $\subset \mathrm{A}$, then $\theta(\mathrm{A})=\theta(\mathrm{A}) \cap \theta(\mathrm{A}) \subset \theta(\theta(\mathrm{A}))$, then by the second property of $\theta$, so $\theta(\theta(\mathrm{A}))=\theta(\mathrm{A})$. A s for the inclusion formula, let $\mathrm{x} \in \theta(\mathrm{W})$, obviously $\mathrm{x} \in \mathrm{W}$, so there is a $\lambda^{\prime} \in \Lambda$ such that $x \in W_{\lambda^{\prime}}$ Then $x$ belongs to $\theta(\mathrm{W}) \cap W_{\lambda^{\prime}} \subset \theta\left(W_{\lambda^{\prime}}\right) \subset$ $\cup_{\lambda \in \Lambda} \theta\left(\mathrm{W}_{\lambda}\right)$ by the second property of $\theta$. Thus $\mathrm{x} \in \theta(\mathrm{W}) \cap\left(\cup_{\lambda^{\prime} \in \Delta^{\prime}} \theta\left(\mathrm{W}_{\lambda^{\prime}}\right)\right) \subset$ $\theta\left(\cup_{\lambda \in \Lambda} \theta\left(\mathrm{W}_{\lambda}\right)\right.$ also follows from the second property of $\theta$.

Definition 2 . Let $\mathrm{G}$ be a non-empty set. A n ordered relation $\leq$ on $\mathrm{G}$ is a binary relation such that for any, $a, b, c \in G$,

(1) $\mathrm{a} \leq \mathrm{a}$,

(2) $a \leq b$ and $b \leq$ cimply $a \leq c$.

Furthermore, $\leq$ is called a preference on $\mathrm{G}$ if

(3) either $a \leq b$ or $b \leq a$ for any $a, b \in G$.

We can identify the binary relation $\leq$ with a subset $\mathrm{g}$ of the Cartesian product $G \times G$, then (1), (2), and (3) can be written as $g \leq 1$, gog $\leq g$, and $g \vee g^{-1}=G \times G$, where $I=\{(a, a) \mid a \in G\}, \wedge, \vee$, and $\leq$ denote the intersection, union, and inclusion of sets respectively, and $g^{-1}=\{(a, b) \mid(b, a) \in g\}$. Note that $g \wedge g^{-1}$ is always an equivalence relation on $\mathrm{G}$. Ordered relations have been generalized to quasi-ordered relation in Chen and $\mathrm{Wu}$ (1984). A relation $\mathrm{g}$ on $\mathrm{G}$ is called quasiordered if $(1) \mathrm{I} \leq \mathrm{g} \wedge \mathrm{g}^{-1},(2)$, $g \circ\left(\mathrm{g}-\mathrm{g}^{-1}\right) \leq \mathrm{g},\left(\mathrm{g}-\mathrm{g}^{-1}\right) \circ \mathrm{g} \leq \mathrm{g}$, and $\left(\mathrm{g}-\mathrm{g}^{-1}\right) \circ\left(\mathrm{g}-\mathrm{g}^{-1}\right)$ $\leq\left(\mathrm{g}-\mathrm{g}^{-1}\right)$. A $\mathrm{n}$ ordered (quasi-ordered) relation $\mathrm{g}$ on $\mathrm{G}$ induces an optimum operator $\theta: P(G) \rightarrow P(G)$ by setting $\theta(W)=\{x \in W \mid$ there is no $a \in W$ such that $\left.(a, x) \in g-g^{-1}\right\}$ for any $W \subset G$.

Definition 3. Let $g$ be an ordered relation on a non-empty set $G$. Given a subset $W$ of $G$, if for any $a \in W-\theta(W)$, there exists $a^{\prime} \in \theta(W)$ such that $\left(a^{\prime}, x\right) \in g-g^{-1}$, then $W$ is said to satisfy G-property.

Theorem 1. Let $g$ be an ordered relation on a non-empty set $G$, and let be $\theta$ be the optimum operator induced by $g$. If a subset $W$ of $G$ satisfies the G-property, then $\theta$ satisfies the global-local principle of optimality on W, namely 


$$
\theta(W)=\theta\left(\bigcup_{\lambda \in \Lambda} \theta\left(W_{\lambda}\right)\right)
$$

Proof. For any $x \in \theta\left(\cup_{\lambda \in \Delta} \theta\left(W_{\lambda}\right)\right)$, then $x \in \cup_{\lambda \in \Delta} \theta\left(W_{\lambda}\right) \subset . \cup_{\lambda \in \Delta} W_{\lambda}$. Suppose that $x \in \theta(W)$, there is an $x^{\prime} \in \theta(W)$ such that $\left(x^{\prime}, x\right) \in g-g^{-1}$ by the $G$-property of $\theta$. Note that there exists $\lambda^{\prime} \in \Lambda$ such that $x^{\prime} \in W_{\lambda^{\prime}}$ then $x^{\prime} \in \theta(W) \cap W_{\lambda^{\prime}} \subset$ $\theta\left(\mathrm{W}_{\lambda^{\prime}}\right) \subset \cup_{\lambda \in \Delta} \theta\left(\mathrm{W}_{\lambda}\right)$. T hus $\left(\mathrm{x}^{\prime}, \mathrm{x}\right) \in \mathrm{g}-\mathrm{g}^{-1}$ is a contradiction to the optimality of $\mathrm{x}$ in $\cup_{\lambda \in \Delta} \theta\left(\mathrm{W}_{\lambda}\right)$. So $\left(\theta \cup_{\lambda \in \Delta} \theta\left(\mathrm{W}_{\lambda}\right)\right) \subset \theta$ (W). The other side follows from Proposition 1.

Transformation principle of optimality: Let $\theta_{1}$ and $\theta_{2}$ be optimum operators on non-empty sets $X$ and $Y$ respectively. Let $f: P(X) \rightarrow P(Y)$ be a mapping. Given a subset of $W$ of $X$, if

$$
\theta_{2}(f(W))=\theta_{2}\left(f\left(\theta_{1}(W)\right)\right.
$$

then $\mathrm{f}, \theta_{1}$, and $\theta_{2}$ are said to satisfy the transformation principle of optimality on $\mathrm{W}$.

This principle is a typical example of projective (or body-shadow) relations $(W u, 1982,1983,1984 a)$. It relates the selection on $f(W)$ to the selection on the part $f\left(\theta_{1}(W)\right)$. Thus, if the principle is satisfied, the number of selections will be reduced by not necessarily considering all the possible choices.

Definition 4. Let $g_{1}$ and $g_{2}$ be ordered relations on non-empty sets $G_{1}$ and $G_{2}$ respectively. Given a function $f: G_{1} \rightarrow G_{2}$, if $(a, b) \in g_{1}-g_{1}^{-1}$ implies that ( $f(a)$, $f(b)) \in g_{2}-g_{2}^{-1}$ for any $a, b \in G_{1}$, then $f$ is called strictly monotonic.

Theorem 2. Let $G_{1}$ and $G_{2}$ be two non-empty sets, and let $f: G_{1} \rightarrow G_{2}$ be a mapping. Let $\theta_{1}$ and $\theta_{2}$ be optimum operators on $G_{1}$ and $G_{2}$ induced by the ordered relations $g_{1}$ on $G_{1}$ and $g_{2}$ on $G_{2}$ respectively. If a subset $W$ of $G_{1}$ satisfies $G_{1}$ - property and $f$ is strictly monotonic, then $f, \theta_{1}$ and $\theta_{2}$ satisfy the transformation principle of optimality on W, namely,

$$
\theta_{2}(f(W))=\theta_{2}\left(f\left(\theta_{1}(W)\right) .\right.
$$

Proof. For any $y \in \theta_{2}(f(W)) \subset f(W)$, there exists an $x \in W$ such that $y=f(x)$. If $x \in \theta_{1}(W)$, then $y=f(x) \in f\left(\theta_{1}(W)\right)$. Thus $y \in \theta_{2}(f(W)) \cap f(\theta,(W)) \subset$ $\theta_{2}\left(f\left(\theta_{1}(W)\right)\right)$ by definition of $\theta_{2}$. If $x \in \theta_{1}(W)$, then there is an $x^{\prime} \in \theta_{1}(W)$ such that $\left(x^{\prime}, x\right) \in g_{1}-g_{1}^{-1}$ by the $G_{1}$-property of $\theta_{1}$. Thus $\left(f\left(x^{\prime}\right), f(x)\right) \in g_{2}-g_{2}^{-1}$ by the strict monotonicity of $f$. Since there is no $y^{\prime \prime} \in f(W)$ such that $\left(y^{\prime \prime}, y\right) \in g_{2}-g_{2}^{-1}$ then $\left(f\left(x^{\prime}\right), f(x)\right) \in-g_{2}^{-1}$. This is a contradiction.

On the other hand, for any $y \in \theta_{2}\left(f_{2}\left(\theta_{1}(W)\right)\right)$, there is an $x \in \theta_{1}(W)$ such that $y=f(x)$. Suppose that $y \in \theta_{2}(f(W))$, then there exists a $y^{\prime} \in f(W)$ such that 
Kybernetes 26,3 $\left(y^{\prime}, y\right) \in g_{2}-g_{2}^{-1}$. Let $x^{\prime}$ be an element of $W$ such that $y^{\prime}=f\left(x^{\prime}\right)$. If $x^{\prime} \in \theta_{1}(W)$, then $\left(y^{\prime}, y\right) \in g_{2}-g_{2}^{-1}$ is a contradiction to the optimality of $y$ in $f\left(\theta_{1}(W)\right)$. If $x^{\prime} \notin \theta_{1}(W)$, there exists an $x^{\prime \prime} \in \theta_{1}(W)$ such that $\left(x^{\prime \prime}, x^{\prime}\right) \in g_{1}-g_{1}^{-1}$ by the $G_{1}$ - property of $g_{1}$. Then $\left(f\left(x^{\prime \prime}\right), f\left(x^{\prime}\right)\right) \in g_{2}-g_{2}^{-1}$. Thus $\left(f\left(x^{\prime \prime}\right), y\right) \in g_{2}-g_{2}^{-1}$, which is also a contradiction to the optimality of $y$ in $f\left(\theta_{1}(W)\right)$.

For quasi-ordered relations given by Chen and Wu (1984), the above theorem is also true.

\section{F undamental equations of optimality}

Generalized dynamic programming

Let $\Delta$ and $\mathrm{W}$ be non-empty sets. Given an optimum operator $\theta$ on W and a mapping $\mathrm{I}: \Delta \rightarrow \mathrm{W}$ which is called a criteria function, a policy is an element $\delta$ of $\Delta$, and its value I $\delta$ ) in W is called the criteria index of $\delta$. We usually denote

$$
\theta-\underset{\delta \in \Delta}{\operatorname{Optim}}\{I(\delta)\}=\theta(I(\Delta))
$$

to be the $\theta$-optimization of I $(\delta)$ over $\Delta$.

Let $\theta$ and $\theta^{\prime}$ be optimum operators on non-empty sets $W$ and $W^{\prime}$ respectively. Given a family of non-empty sets $\Delta(\gamma)(\gamma \in \Gamma)$ and two mappings

$$
\begin{gathered}
F: \Gamma \times W \longrightarrow W^{\prime}, \\
y: \Delta(\Gamma) \longrightarrow W,
\end{gathered}
$$

the set $\Delta(\Gamma)=\cup_{\gamma \in \Gamma} \gamma_{\times} \Delta(\gamma)$ is called the policy set, and each element of $\Delta(\Gamma)$ is called a policy. For each policy $(\gamma, \delta) \in \Delta(\Gamma), \delta$ is called the sub-policy of $(\gamma, \delta)$. For each $\gamma \in \Gamma$, let

$$
\begin{aligned}
Y_{\gamma} & =\left\{y(\gamma, \delta) \in W^{\prime} \mid \delta \in \Delta(\gamma)\right\}, \\
Z_{\gamma} & =F\left(\gamma \times Y_{\gamma}\right)=\left\{F(\gamma, y) \mid y \in Y_{\gamma}\right\} \\
& =\{F(\gamma, y(\gamma, \delta)) \mid \delta \in \Delta(\gamma)\} .
\end{aligned}
$$

Then

$$
\begin{aligned}
\bigcup_{\gamma \in \Gamma} Z_{\gamma} & =\left\{F(\gamma, y) \mid \gamma \in \Gamma, y \in Y_{\gamma}\right\} \\
& =\{F(\gamma, y(\gamma, \delta)) \mid \gamma \in \Gamma, \delta \in \Delta(\gamma)\} \\
& =\{F(\gamma, y(\gamma, \delta)) \mid(\gamma, \delta) \in \Delta(\Gamma)\} .
\end{aligned}
$$


Thus

$$
\begin{aligned}
& \underset{\delta \in \Delta(\gamma)}{\theta^{\prime}-O \operatorname{ptim}}\{F(\gamma, y(\gamma, \delta))\}=\theta^{\prime}\left(Z_{\gamma}\right), \\
& \underset{\gamma \in \Gamma, \delta \in \Delta(\gamma)}{\theta^{\prime}-\text { Optimim }}\{F(\gamma, y(\gamma, \delta))\}=\theta^{\prime}\left(\bigcup_{\gamma \in \Gamma} Z_{\gamma}\right), \\
& \underset{\delta \in \Delta(\gamma, y)}{\theta^{\prime}-O p \operatorname{sim}}\{F(\gamma, \theta-O \operatorname{Optim}\{y(\gamma, \delta)\})\}=\theta^{\prime}\left(F\left(\gamma, \theta\left(Y_{\gamma}\right)\right)\right) \text {, } \\
& \underset{\gamma \in \Gamma, \delta \in \Delta(\gamma, y)}{\theta^{\prime}-O p t i m}\left\{\theta_{\delta \in \Delta(\gamma)}\{F(\gamma, y(\gamma, \delta))\}\right\}=\theta^{\prime}\left(\bigcup_{\gamma \in \Gamma} \theta^{\prime}\left(F\left(\gamma, Y_{\gamma}\right)\right)\right) \text {, }
\end{aligned}
$$

and where

$$
\begin{aligned}
\gamma \times \Delta(\gamma, y) & =(\gamma \times \Delta(\gamma)) \cap y^{-1} \theta y(\gamma \times \Delta(\gamma)) \\
\gamma \times \Delta(\gamma, F) & =(\gamma \times \Delta(\gamma)) \cap y^{-1} p F^{-1} \theta^{\prime} F(\gamma \times y(\gamma \times \Delta(\gamma))) \\
\gamma \times \Delta(\gamma, y, F) & =(\gamma \times \Delta(\gamma, y)) \cap y^{-1} p F^{-1} \theta^{\prime} F(\gamma \times y(\Delta(\gamma, y))),
\end{aligned}
$$

where $\rho$ is the canonical projection from $\Gamma \times \mathrm{W}$ to W. If $\theta^{\prime}$ satisfies the globallocal principle of optimality, and $F, \theta, \theta^{\prime}$ satisfy the transformation principle of optimality, then

$$
\theta^{\prime}\left(\bigcup_{\gamma \in \Gamma} F\left(\gamma, Y_{\gamma}\right)\right)=\theta^{\prime}\left(\bigcup_{\gamma \in \Gamma} \theta^{\prime} F\left(\gamma, \theta\left(Y_{\gamma}\right)\right)\right)
$$

Conservation principle of optimality: In a generalized dynamic programming model, if the equation (3) holds, then the model is said to satisfy conservation principle of optimality.

Theorem 3. For generalized dynamic programming, the fundamental equation

$$
\begin{aligned}
\underset{\gamma \in \Gamma, \delta \in \Delta(\gamma)}{\theta^{\prime}-O p \operatorname{tim}}\{F(\gamma, y(\gamma, \delta))\}= & \underset{\substack{\theta^{\prime}-O p \operatorname{Optim} \delta \in \Delta(\gamma, y, F) \\
\gamma \in \Delta(\gamma, y)}}{\left\{\begin{array}{c}
\theta^{\prime} \text {-Optim } \\
\delta \in \Delta(\gamma)
\end{array}\right.} \\
& {[F(\gamma, \theta-O p \operatorname{tim}\{y(\gamma, \delta)\})]\} }
\end{aligned}
$$

holds if and only if the conservation principle of optimality is satisfied.

Proof. For the convenience of our discussion, we introduce the following notations: 
Kybernetes

26,3

322

$$
\begin{aligned}
\Delta(\Gamma, y):= & \bigcup_{\gamma \in \Gamma} \gamma \times \Delta(\gamma, y) \\
\Delta(\Gamma, F):= & \Delta(\Gamma) \cap y^{-1} p F^{-1} \theta^{\prime} F(\Gamma \times y(\Delta(\Gamma))) \\
\Delta(\Gamma, y, F):= & \left(\bigcup_{\gamma \in \Gamma} \gamma \times \Delta(\gamma, y, F)\right) \cap \\
& y^{-1} p F^{-1} \theta^{\prime} F\left(\bigcup_{\gamma \in \Gamma} \gamma \times y(\gamma \times \Delta(\gamma, y, F))\right) .
\end{aligned}
$$

Then

$$
\begin{aligned}
& \underset{\gamma \in \Gamma, \delta \in \Delta(\gamma)}{\theta^{\prime}-O \operatorname{ptim}}\{F(\gamma, y(\gamma, \delta))\}=\theta^{\prime}\{F(\gamma, y(\gamma, \delta)) \mid(\gamma, \delta) \in \Delta(\Gamma)\} \\
& =\theta^{\prime}\{F(\gamma, y(\gamma, \delta)) \mid \gamma \in \Gamma, \delta \in \Delta(\gamma, F)\} \\
& =\theta^{\prime}\left(\bigcup_{\gamma \in \Gamma} \theta^{\prime} F(\gamma, \theta\{y(\gamma, \delta) \mid \delta \in \Delta(\gamma)\})\right) \\
& =\theta^{\prime}\left(\bigcup_{\gamma \in \Gamma} \theta^{\prime}\{F(\gamma, y(\gamma, \delta)) \mid \delta \in \Delta(\gamma, y)\}\right) \\
& =\theta^{\prime}\{F(\gamma, y(\gamma, \delta)) \mid \gamma \in \Gamma, \delta \in \Delta(\gamma, y, F)\} \\
& =\{F(\gamma, y(\gamma, \delta)) \mid(\gamma, \delta) \in \Delta(\Gamma, y, F)\} \\
& =\underset{\gamma \in \Gamma, \delta \in \Delta(\gamma, y, F)}{\theta^{\prime}-\operatorname{pptim}}\left\{\underset{\delta \in \Delta(\gamma, y)}{\theta^{\prime}-\mathrm{O}_{\mathrm{ptim}}}\left[F\left(\gamma, \theta-\operatorname{Optim}_{\delta \in \Delta(\gamma)}\{y(\gamma, \delta)\}\right)\right]\right\}
\end{aligned}
$$

Proposition 2. In any generalized dynamic programming model, we have the following relations:

$$
\begin{aligned}
& \Delta(\gamma, y) \cap \Delta(\gamma, F) \subset \Delta(\gamma, y, F) \subset \Delta(\gamma), \quad \forall \gamma \in \Gamma \\
& \Delta(\Gamma, y) \cap \Delta(\Gamma, F) \subset \Delta(\Gamma, y, F) \subset \Delta(\Gamma) .
\end{aligned}
$$

Proof. Let $\delta^{*} \in \Delta,(\gamma, \mathrm{F})$, note that $\mathrm{F}\left(\gamma, \mathrm{y}\left(\gamma, \delta^{*}\right)\right) \in \theta^{\prime}\{\mathrm{F}(\gamma, \mathrm{y}(\gamma, \delta)) \mid \delta \in$ $\Delta(\gamma)\}$. If $\delta^{*} \in \Delta(\gamma, \mathrm{y})$, then $\mathrm{F}\left(\gamma, \mathrm{y}\left(\gamma, \delta^{*}\right)\right) \in\{\mathrm{F}(\gamma, \mathrm{y}(\gamma, \delta)) \mid \delta \in \Delta(\gamma, \mathrm{y})\}$. Thus $\mathrm{F}\left(\gamma, \mathrm{y}\left(\gamma, \delta^{*}\right)\right) \in \theta^{\prime}\{\mathrm{F}(\gamma, \mathrm{y}(\gamma, \delta)) \mid \delta \in \Delta(\gamma, \mathrm{y})\}$ by the definition of the optimum operator $\theta^{\prime}$. So $\delta^{*} \in \Delta(\gamma, \mathrm{y}, \mathrm{F})$ by the definition of $\Delta(\gamma, \mathrm{y}, \mathrm{F})$.

Similarly, for any $\left(\gamma^{*}, \delta^{*}\right) \in \Delta(\Gamma, \mathrm{F})$, it follows that

$$
F\left(\gamma^{*}, y\left(\gamma^{*}, \delta^{*}\right)\right) \in\{F(\gamma, y(\gamma, \delta)) \mid \gamma \in \Gamma, \delta \in \Delta(\gamma, y) \text {. }
$$


If $\left(\gamma^{*}, \delta^{*}\right) \in \Delta(\Gamma, \mathrm{y})$, then

$$
F\left(\gamma^{*}, y\left(\gamma^{*}, \delta^{*}\right)\right) \in\{F(\gamma, y(\gamma, \delta)) \mid \gamma \in \Gamma, \delta \in \Delta(\gamma, y) .
$$

Thus

$$
F\left(\gamma^{*}, y\left(\gamma^{*}, \delta^{*}\right)\right) \in \theta^{\prime}\{F(\gamma, y(\gamma, \delta)) \mid \gamma \in \Gamma, \delta \in \Delta(\gamma, y) .
$$

The above set is also contained in $\theta^{\prime}\left(\cup_{\gamma \varepsilon \Gamma} \theta^{\prime}\{F(\gamma, y(\gamma, \delta)) \mid \in \Delta(\gamma, y)\}\right)$ by Proposition 1. Therefore $\left(\gamma^{*}, \delta^{*}\right) \in \Delta(\Gamma, \mathrm{y}, \mathrm{F})$

Corollary 1 . If $\theta^{\prime}$ satisfies the global-local principle of optimality, and $F, \theta, \theta^{\prime}$ satisfy the transformation principle of optimality, then the fundamental equation (4) holds.

Proof. Since

$$
\begin{gathered}
\theta^{\prime}\left(\bigcup_{\gamma \in \Gamma} Z_{\gamma}\right)=\theta^{\prime}\left(\bigcup_{\gamma \in \Gamma} \theta^{\prime}\left(Z_{\gamma}\right)\right), \\
\theta^{\prime}\left(Z_{\gamma}\right)=\theta^{\prime}\left(F\left(\gamma, Y_{\gamma}\right)\right)=\theta^{\prime}\left(F\left(\gamma, \theta\left(Y_{\gamma}\right)\right)\right),
\end{gathered}
$$

then

$$
\theta^{\prime}\left(\bigcup_{\gamma, \Gamma} F\left(\gamma, Y_{\gamma}\right)\right)=\theta^{\prime}\left(\bigcup_{\gamma \in \Gamma} \theta^{\prime}\left(F\left(\gamma, \theta\left(Y_{\gamma}\right)\right)\right)\right),
$$

and this means that the conservation principle of optimality is satisfied.

Definition 5. A n optimal policy $(\gamma, \delta)$ in a generalized dynamic programming model is said to satisfy the Bellman principle of optimality if its sub-policy $\delta$ is optimal. If all the optimal policies satisfy the Bellman principle of optimality, then the model is said to satisfy the Bellman principle of optimality.

Definition 6. For an optimization problem, if it can be formulated as a dynamic programming model for which $\Delta(\Gamma, F)=\Delta(\Gamma, y, F)$, then the problem is called solvable by dynamic programming.

In the above analysis of the fundamental equation, we know that any optimal policy that satisfies the Bellman principle of optimality belongs to $\Delta(\Gamma, \mathrm{y}, \mathrm{F})$ regardless of whether or not the fundamental equation (4) is held. We summarize our conclusions as follows.

(1) The satisfaction of the Bellman principle of optimality in a dynamic programming model implies

$$
\Delta(\Gamma, F) \subset \Delta(\Gamma, y, F) \text {. }
$$


Kybernetes 26,3

324
In fact $\Delta(\Gamma, F) \cup \Delta(\Gamma, y, F)$, is the set of all optimal policies that satisfy the Bellman principle of optimality for any dynamic programming model.

(2) The holding of the fundamental equation in a dynamic programming model implies

$$
\Delta(\Gamma, y, F) \subset \Delta(\Gamma, F),
$$

that is, all the solutions of fundamental equation (4) are optimal policies of the model, and they satisfy the Bellman principle of optimality.

(3) The satisfaction of the Bellman principle of optimality and the holding of the fundamental equation imply:

$$
\Delta(\Gamma, F)=\Delta(\Gamma, y, F) .
$$

(4) The satisfaction of the Bellman principle of optimality cannot guarantee the holding of the fundamental equation. Conversely, the holding of the fundamental equation does not imply the satisfaction of the Bellman principle of optimality. There are examples for both cases (Hu, 1982).

(5) A problem can be solved by the dynamic programming method if and only if it can be formulated as a dynamic programming model for which the Bellman principle of optimality and the conservation principle of optimality are simultaneously satisfied.

\section{Deterministic discrete model}

We consider a general sequential $n$-stage decision system in which the stages are denoted $0,1,2 \cdots, n$. A t each stage $k$ there is a state set $X_{k}$, the elements of $X_{k}$ are called states at $k$. For each state $x_{k}$ of $X_{k}$ there is a decision set $U\left(x_{k}\right)$, the elements of $U\left(x_{k}\right)$ are called decisions at $x_{k}$, and $U_{k}=\cup_{x_{k}} \in X_{k} U\left(x_{k}\right)$ is called the decision set at stage $k$.

Let the state transition equation be given by

$$
x_{k+1}=T_{k}\left(x_{k}, u_{k}\right)
$$

where $x_{k}$ varies in $X_{k}$ and $u_{k}$ varies in $U\left(x_{k}\right)$, so $T_{k}$ is a map

$$
T_{k}: \bigcup_{x_{k} \in X_{k}} x_{k} \times U\left(x_{k}\right) \longrightarrow X_{k+1} \text {. }
$$

The policy set of the decision system is defined to be

$$
\begin{aligned}
\Delta= & \left\{\delta=\left(u_{0}, u_{1}, \cdots, u_{n-1}\right) \mid u_{k} \in U\left(x_{k}\right),\right. \\
& \left.x_{k+1}=T_{k}\left(x_{k}, u_{k}\right), k=0,1, \cdots, n-1\right\},
\end{aligned}
$$


and the elements of $\Delta$ are called policies. For a state $x_{k}$ of $X_{k}$, we define the sub-policy set $\Delta\left(\mathrm{x}_{\mathrm{k}}\right)$ at the state $\mathrm{x}_{\mathrm{k}}$ as follows:

$$
\begin{aligned}
\Delta\left(x_{k}\right)= & \left\{\delta=\left(u_{k}, u_{k+1}, \cdots, u_{n-1}\right) \mid u_{i} \in U\left(x_{i}\right),\right. \\
& \left.x_{i+1}=T_{i}\left(x_{i}, u_{i}\right), i=k, k+1, \cdots, n-1\right\} .
\end{aligned}
$$

A criteria index function $V_{k}$, which is defined as a judgement of elements of optimization $\Delta_{k}=\cup x_{k} \in X_{k} \Delta\left(x_{k}\right)$ in a criteria set $W_{k^{\prime}}$ can be viewed as a map

$$
V_{k}: \prod_{i=k}^{n-1}\left(\bigcup_{x_{i} \in X_{i}} x_{i} \times U\left(x_{i}\right)\right) \rightarrow W_{k} .
$$

The optimum operator on $\mathrm{W}_{\mathrm{k}}$ is denoted by $\theta_{\mathrm{k}}, 0 \leq \mathrm{k} \leq \mathrm{n}-1$.

In order to derive the fundamental equation similar to equation (4), we need the following transformation $F_{k}$ which turns out the criteria index in $W_{k}$ for each state $x_{k}$ and each decision $u_{k} \in U x_{k}$ ), that is,

$$
F_{k}:\left(\bigcup_{x_{i} \in X_{i}} x_{i} \times U\left(x_{i}\right)\right) \times W_{k+1} \longrightarrow W_{k} .
$$

Let $p_{k}$ denote the canonical projection from $\left(\cup_{x_{i} \in X_{i}} x_{i} \times U\left(x_{i}\right)\right) \times W_{k+1}$ to $W_{k+1}$. We need to assume that $V_{k}$ satisfies the following recursive relation:

$$
\begin{aligned}
& V_{k}\left(x_{k}, u_{k} ; \cdots ; x_{n-1}, u_{n-1}\right) \\
& =F_{k}\left(x_{k}, u_{k} ; V_{k+1}\left(x_{k+1}, u_{k+1} ; \cdots ; x_{n-1}, u_{n-1}\right)\right)
\end{aligned}
$$

where $\mathrm{x}_{\mathrm{i}+1}=\mathrm{T}_{\mathrm{i}}\left(\mathrm{x}_{\mathrm{i}}, \mathrm{u}_{\mathrm{i}}\right), \mathrm{k} \leq \mathrm{i} \leq \mathrm{n}-1$. Note that $\delta_{\mathrm{k}}=\left(\mathrm{u}_{\mathrm{k}} ; \cdots ; \mathrm{x}_{\mathrm{n}-1}, \mathrm{u}_{\mathrm{n}-1}\right)$ belongs to $\Delta\left(x_{k}\right)$, and its components can be viewed as the variables, $u_{k}, \cdots, x_{n-1}, u_{n-1}$ such that $u_{i} \in U\left(x_{i}\right), k \leq i \leq n-1$. T hen $v_{k}$ becomes a function of variables $x_{k}$ and $\delta_{k}$. Let the new criteria function be denoted by $I_{k}$, that is

$$
I_{k}\left(x_{k}, \delta_{k}\right)=V_{k}\left(x_{k}, u_{k} ; \cdots ; x_{n-1}, u_{n-1}\right) .
$$

Thus the recursive equation (14) becomes

$$
I_{k}\left(x_{k}, u_{k}\right)=F_{k}\left(x_{k}, u_{k} ; I_{k+1}\left(x_{k+1}, \delta_{k+1}\right)\right) .
$$

If $x_{k+1}=T_{k}\left(x_{k}, u_{k}\right)$ is substituted into the recursive equation (15), we can identify the system between stage $k$ and stage $k+1$ as the fundamental prototype of the last section. More specifically, we set $\left(x_{k}, u_{k}\right)=\gamma_{1} x_{k} \times U\left(x_{k}\right)=$ $\Gamma, \Delta\left(\mathrm{T}_{\mathrm{k}}\left(\mathrm{x}_{\mathrm{k}^{\prime}} \mathrm{u}_{\mathrm{k}}\right)\right)=\Delta(\gamma), \mathrm{W}_{\mathrm{k}+1}=\mathrm{W}, \mathrm{W}_{\mathrm{k}}=\mathrm{W}^{\prime}, \theta_{\mathrm{k}+1}=\theta, \theta_{\mathrm{k}}=\theta^{\prime}, \mathrm{F}_{\mathrm{k}}=\mathrm{F}, \mathrm{I}_{\mathrm{k}+1}=\mathrm{y}$. Then $I_{k+1}$ should be understood by 
Kybernetes

26,3

$$
I_{k+1}\left(x_{k}, u_{k}, T_{k}\left(x_{k}, u_{k}\right), \delta_{k+1}\right)=I_{k+1}\left(T_{k}\left(x_{k}, u_{k}\right), \delta_{k}\right) .
$$

Under this identification, we have

$$
\Delta\left(x_{k}, u_{k}, I_{k+1}\right)=\Delta(\gamma, y)
$$

\section{6}

$\Delta\left(x_{k}, I_{k+1}, F_{k}\right)=\Delta(\Gamma, y, F)$.

Theorem 4. If each optimum operator $\theta_{k}$ and each map $F_{k}$ satisfies the global-local principle and transformation principle of optimality, then we have the following fundamental recursive equation at each state $x_{k} \in X_{k}$ :

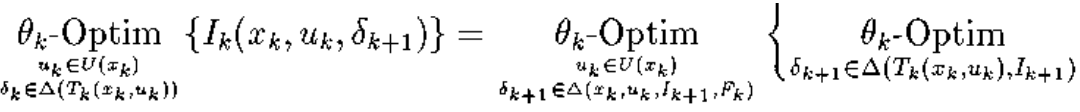

$$
\begin{aligned}
& \left.\left[F_{k}\left(x_{k}, u_{k}, \underset{\substack{\theta_{k+1}-\operatorname{Optim} \\
\delta_{k+1} \in \Delta\left(T_{k}\left(x_{k}, u_{k}\right)\right)}}{\theta_{k+1}}\left\{I_{k+1}\left(T_{k}\left(x_{k}, u_{k}\right), \delta_{k+1}\right)\right\}\right)\right]\right\} \text {. }
\end{aligned}
$$

Proposition 3 . In any generalized dynamic programming model, the following relations are valid for any $x_{k}$ and $u_{k} \in U\left(x_{k}\right)(k=0,1, \cdots, n-1)$ :

$$
\begin{gathered}
\Delta\left(x_{k}, u_{k}, I_{k+1}\right) \cap \Delta\left(x_{k}, u_{k}, F_{k}\right) \subset \Delta\left(x_{k}, u_{k}, I_{k+1}, F_{k}\right) \subset \Delta\left(T_{k}\left(x_{k}, u_{k}\right)\right), \\
\Delta\left(x_{k}, I_{k+1}\right) \cap \Delta\left(x_{k}, F_{k}\right) \subset \Delta\left(x_{k}, I_{k+1}, F_{k}\right) \subset \Delta\left(x_{k}\right) .
\end{gathered}
$$

The advantage of the generalized recursive equation (16) is that $\theta_{k}$ may be deductively determined to satisfy the conditions of Theorem 4 or Theorem 3. 
Thus some problems that can not be formulated as ordinary dynamic programming problems might be solved with generalized dynamic programming by constructing certain appropriate optimum operators. The following example demonstrates such a possibility.

\section{Example}

Consider the multistage sequential decision system illustrated by Figure 1. The problem is to search for the $\bmod (4)$-shortest paths from $x_{0}$ to $x_{4}$, namely, the paths whose lengths have the minimal remainder modulo 4 , the smallest number of $\{0,1,3,4\}$.

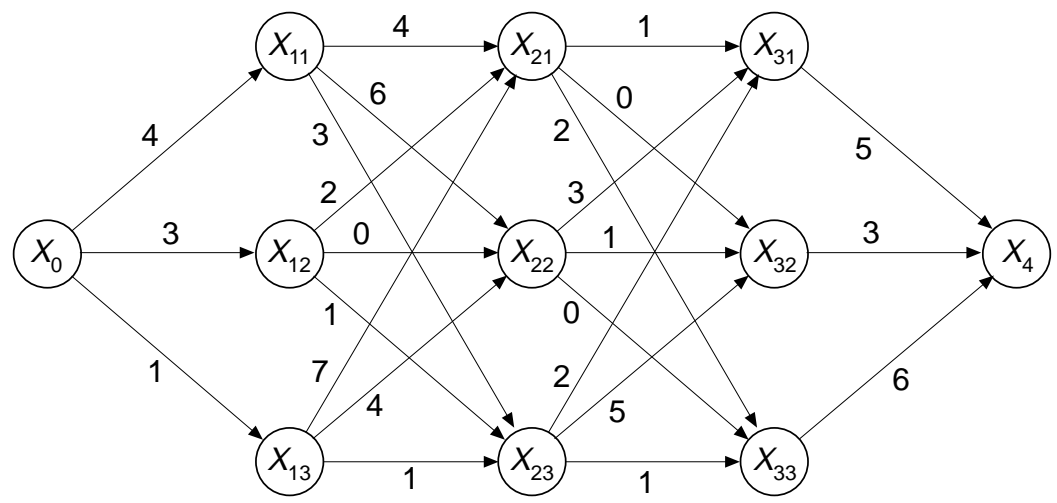

Figure 1.

In this problem, it can be easily seen that the policy $x_{0} \rightarrow x_{12} \rightarrow x_{21} \rightarrow x_{32} \rightarrow$ $x_{4}$ is optimal, while its sub-policy $x_{21} \rightarrow x_{33} \rightarrow x_{4}$ is not optimal since its length is 3 and the path $x_{21} \rightarrow x_{33} \rightarrow x_{4}$ has length 0 modulo 4 . The Bellman principle of optimality is thus not valid for the optimum operator $\operatorname{Minmod}(4): \mathbf{P}(\mathbf{N}) \rightarrow$ $\mathbf{P}(\mathbf{N})$, defined by:

$$
\operatorname{Minmod}(4)(A)=\operatorname{Min}\{k \mid 4 r+k \in A, r, k \in \mathbf{N}\}
$$

for nonempty subsets $A$ of the set $\mathbf{N}$ of nonnegative integers. Since $\theta_{0}$ is Minmod (4), then $\theta_{1}$ is Mod (4) which is defined by

$$
\operatorname{Mod}(4)(A)=\{k \mid 4 r+k \in A, r, k \in \mathbf{N}, 0 \leq k \leq 4\}
$$

for non-empty subsets $A$ of $\mathbf{N} \theta_{2}$ and $\theta_{3}$ can be similarly determined, and $\theta_{1}$ $=\theta_{2}=\theta_{3}=\operatorname{Mod}(4)$.

Ât stage 3:

$$
\begin{array}{ll}
x_{31}: & \{5\}=\{1\}(\bmod 4), \\
x_{32}: & \{\underline{3}\}=\{\underline{3}\}(\bmod 4), \\
x_{33}: & \{\underline{6}\}=\{\underline{2}\}(\bmod 4) .
\end{array}
$$


Kybernetes

26,3

328
At stage 2:

$x_{21}$ :

$[\{1\},\{0\},\{2\}]+[\{1\},\{\underline{3}\},\{\underline{2}\}]$

$=[\{2\},\{3\},\{\underline{4}\}]$

$=\{\underline{0}, 2, \underline{3}\}(\bmod 4)$,

$[\{3\},\{1\},\{0\}]+[\{1\},\{3\},\{\underline{2}\}]$

$=[\{4\},\{4\},\{\underline{2}\}]$

$=\{0, \underline{2}\}(\bmod 4)$,

$x_{23}$ :

$[\{2\},\{5\},\{1\}]+[\{1\},\{\underline{3}\},\{2\}]$

$=[\{3\},\{\underline{8}\},\{3\}]$

$=\{\underline{0}, 3\}(\bmod 4)$.

At stage 1:

$x_{11}$ :

$[\{4\},\{6\},\{3\}]+[\{\underline{0}, 2,3\},\{0, \underline{2}\},\{0,3\}]$

$=[\{\underline{4}, 6,7\},\{6, \underline{8}\},\{3,6\}]$

$=\{\underline{0}, 2,3\}(\bmod 4)$,

$x_{12}: \quad[\{2\},\{0\},\{1\}]+[\{0,2, \underline{3}\},\{0,2\},\{\underline{0}, 3\}]$

$=[\{2,4, \underline{5}\},\{0,2\},\{\underline{1}, 4\}]$

$=\{0, \underline{1}, 2\}(\bmod 4)$,

$x_{13}: \quad[\{7\},\{4\},\{1\}]+[\{\underline{0}, 2,3\},\{0,2\},\{0,3\}]$

$=[\{\underline{z}, 9,10\},\{4,6\},\{1,4\}]$

$=\{0,1,2, \underline{3}\}(\bmod 4)$.

At stage 0:

$x_{0}$ :

$[\{4\},\{3\},\{1\}]+[\{\underline{0}, 2,3\},\{0, \underline{1}, 2\},\{0,1,2, \underline{3}\}]$

$=[\{\underline{4}, 6,7\},\{3, \underline{4}, 5\},\{1,2,3, \underline{4}\}]$

$=\{\underline{0}, 1,2,3\}(\bmod 4)$. 
Now, we know that the shortest mod(4)-length is 0 , and the corresponding optimal paths, which can be obtained by tracing some special values back to state $x_{4}$, are as follows:

$$
\begin{aligned}
& x_{0} \longrightarrow x_{11} \longrightarrow x_{21} \longrightarrow x_{33} \longrightarrow x_{4}, \\
& x_{0} \longrightarrow x_{11} \longrightarrow x_{22} \longrightarrow x_{33} \longrightarrow x_{4}, \\
& x_{0} \longrightarrow x_{12} \longrightarrow x_{21} \longrightarrow x_{32} \longrightarrow x_{4} \\
& x_{0} \longrightarrow x_{12} \longrightarrow x_{23} \longrightarrow x_{32} \longrightarrow x_{4}, \\
& x_{0} \longrightarrow x_{13} \longrightarrow x_{21} \longrightarrow x_{33} \longrightarrow x_{4} .
\end{aligned}
$$

The recursive equation of generalized dynamic programming provides a convenient and effective algorithm for some optimization problems, and its complexity of computation is always a polynomial time. Next, we introduce a matrix multiplication instead of the recursive equation to reduce the complexity of computation. This method is a further development of the work of Qin (1981) and Wu (1984b).

Consider an $n$-stage sequential decision system with stages $0,1,2, \cdots, n$. Let $x_{k}$ be the feasible state set at stage $k(k=1,2, \cdots, n)$, and let the control policy set, which transfers the state $x_{k-1}$ from stage $k-1$ to the state $x_{k}$ at stage $k$, be denoted by $U\left(x_{k-1}, x_{k}\right)(k=1,2, \cdots, n)$. For a policy $u\left(x_{k-1}, x_{k}\right) \in U\left(x_{k-1}, x_{k}\right)$, there is a generalized "return" function $I_{k}\left(u\left(x_{k-1}, x_{k}\right)\right)$ that takes values in a criteria index set $W_{k-1, k^{\prime}}$ that is, a map $I_{k}: U\left(x_{k-1}, x_{k}\right) \rightarrow W_{k-1, k}$. Thus, for the state sets $X_{k-1}$ and $X_{k}(k=1,2, \cdots, n)$, there is a generalized "return" map $A_{k-1, k}: X_{k-1} \times$ $X_{k} \rightarrow P\left(W_{k-1, k}\right)$, which is defined by:

$$
A_{k-1, k}\left(x_{k-1}, x_{k}\right)=I_{k}\left(U\left(x_{k-1}, x_{k}\right)\right), \forall\left(x_{k-1}, x_{k}\right) \in X_{k-1} \times X_{k}
$$

$A_{k-1, k}\left(x_{k-1}, x_{k}\right)$ is the set of return values in $W_{k-1, k}$ If $A_{k-1, k}\left(x_{k-1}, x_{k}\right)=\varnothing$, then $U\left(x_{k-1}, x_{k}\right)=\varnothing$, and this means that the state $x_{k-1}$ can not be transferred to $x_{k}$. For $\mathrm{X}_{0}$ and $\mathrm{X}_{\mathrm{k}}$, we can define a policy map

$$
A_{0, k}: X_{0} \times X_{k} \longrightarrow \mathcal{P}\left(W_{0, k} \times W_{1,2} \cdots \times \times W_{k-1, k}\right)
$$

by

$$
A_{0, k}\left(x_{0}, x_{k}\right)=\bigcup_{x_{1} \in X_{1}, \cdots, x_{k} \in X_{k}} A_{0, k}\left(x_{0}, x_{1}\right) \times \cdots \times A_{0, k}\left(x_{k-1}, x_{k}\right),
$$

for $\left(x_{0}, x_{k}\right) \in X_{0} \times X_{k}$. For each element of $W_{0,1} \times W_{1,2} \times \cdots \times W_{k-1, k^{\prime}}$ we assign a uniform value in a criteria index set $W_{0, k}$, so we have the combined return map

$$
I_{k}: W_{0,1} \times W_{1,2} \times \cdots \times W_{k-1, k} \longrightarrow W_{0, k} .
$$


Kybernetes 26,3

For the criteria index sets $W_{0, k-1}$ and $W_{k-1, k}$ we need to give another combined return map

$$
\oplus_{k-1, k}: W_{0, k-1} \times W_{k-1, k} \longrightarrow W_{0, k} .
$$

The optimum operator on $\mathrm{W}_{0, \mathrm{k}}$ is denoted by $\theta_{\mathrm{k}^{\prime}} \mathrm{k}=1,2, \ldots, \mathrm{n}$.

Theorem 5. If each $\theta_{k}$ satisfies the global-local principle of optimality, and each $I_{k}$ satisfies the transformation principle of optimality, then

$$
\begin{aligned}
\theta_{k}\left(I_{k}\left(A_{0, k}\right)\right)= & \theta_{k}\left[\bigcup _ { x _ { k - 1 } \in X _ { k - 1 } } \theta _ { k } \oplus _ { k - 1 , k } \left(\left(\theta_{k-1} I_{k-1} A_{0, k-1}\left(x_{0}, x_{k-1}\right)\right)\right.\right. \\
& \left.\left.\times A_{k-1, k}\left(x_{k-1}, x_{k}\right)\right)\right] .
\end{aligned}
$$

If we denote $\oplus_{k-1, k}(A \times B)$ by $A \oplus_{k-1} B$ and expand the right side of equation (22), then we have

$$
\begin{aligned}
\theta_{k}\left(I_{k}\left(A_{0, k}\right)\right)= & \theta_{k}\left\{\bigcup _ { x _ { k - 1 } \in X _ { k - 1 } } \theta _ { k - 1 } \left[\bigcup_{x_{k-2} \in X_{k-2}} \ldots\right.\right. \\
& \theta_{2}\left(\bigcup_{x_{1} \in X_{1}}\left(\theta_{1} A_{0,1}\left(x_{0}, x_{1}\right)\right) \oplus_{1,2}\left(A_{1,2}\left(x_{1}, x_{2}\right)\right)\right) \\
& \left.\left.\oplus_{2,3^{\cdots}} \cdots \oplus_{k-2, k-1} A_{k-2, k-1}\right] \oplus_{k-1, k}\left(x_{k-1}, x_{k}\right)\right\}
\end{aligned}
$$

In order to have a similar matrix multiplication as in Qin (1981), we introduce the following binary operation

$$
\odot_{1}: \mathcal{P}\left(W_{0,1}\right)^{X_{0} \times X_{1}} \times \mathcal{P}\left(W_{1,2}\right)^{X_{1} \times X_{2}} \longrightarrow \mathcal{P}\left(W_{1,2}\right)^{X_{0} \times X_{2}}
$$

by

$$
\left(A_{0,1} \odot_{1} A_{1,2}\right)\left(x_{0}, x_{2}\right)=\theta_{2}\left(\bigcup_{x_{1} \in X_{1}}\left(\theta_{1} I_{1} A_{0,1}\left(x_{0}, x_{1}\right)\right) \oplus_{1,2} A_{1,2}\left(x_{1}, x_{2}\right)\right)
$$

for $\left(x_{0} x_{2}\right) \in X_{0} \times X_{2}$. Let $M_{2}=A_{0,1} \odot_{1} A_{1,2}$. For $M_{2}$ and $A_{2,3}$, we similarly define binary operation

$$
\odot_{2}: \mathcal{P}\left(W_{0,2}\right)^{X_{0} \times X_{2}} \times \mathcal{P}\left(W_{2,3}\right)^{X_{2} \times X_{3}} \longrightarrow \mathcal{P}\left(W_{0,3}\right)^{X_{0} \times X_{3}}
$$

by

$$
\left(M_{2} \odot_{2} A_{2,3}\right)\left(x_{0}, x_{3}\right)=\theta_{3}\left(\bigcup_{x_{2} \in X_{2}} M_{2}\left(x_{0}, x_{2}\right) \oplus_{2,3} A_{2,3}\left(x_{2}, x_{3}\right)\right),
$$

for $\left(x_{0}, x_{3}\right) \in X_{0} \times X_{3}$. Inductively, we define $M_{k-1}=M_{k-2} \odot_{k-2} A_{k-2, k-1}$. For $M_{k-1}$ and $A_{k}$, we define 


$$
\bigodot_{k-1}: \mathcal{P}\left(W_{0, k-1}\right)^{X_{0} \times X_{k-1}} \times \mathcal{P}\left(W_{k-1, k}\right)^{X_{k-1} \times X_{k}} \longrightarrow \mathcal{P}\left(W_{0, k}\right)^{X_{0} \times X_{k}}
$$

by

$$
\begin{aligned}
& \left(M_{k-1} \odot_{k-1} A_{k-1, k}\right)\left(x_{0}, x_{k}\right)= \\
& \quad \theta_{k}\left(\bigcup_{x_{k-1} \in X_{k-1}} M_{k-1}\left(x_{0}, x_{k-1}\right) \oplus k-1, k A_{k-1, k}\left(x_{k-1}, x_{k}\right)\right),
\end{aligned}
$$

for $\left(x_{0}, x_{k}\right) \in X_{0} \times X_{k}$. Let us denote $M_{k}=M_{k-1} \odot M_{k-1} A_{k^{\prime}} k=3,4, \ldots, n$. We summarize the above discussion in the following theorem.

Theorem 6. If $\theta_{k}$ and $\theta_{k-1}(k=2,3, \ldots n)$ satisfies the equation (22), then the fundamental equation (16) becomes the matrix multiplication

$$
\begin{array}{r}
M_{k}=\left(\left(\left(A_{0,1} \odot_{1} A_{1,2}\right) \odot_{2} A_{2,3}\right) \odot_{3} \cdots \odot_{k-2} A_{k-2, k-1}\right) \odot_{k-1} A_{k-1, k}, \\
\theta_{k}\left(I_{k} A_{0, k}\left(x_{0}, x_{k}\right)\right)=M_{k}\left(x_{0}, x_{k}\right), \quad\left(x_{0}, x_{k}\right) \in X_{0} \times X_{k} .
\end{array}
$$

In practical problems, one can choose a uniform criteria index set $W$ so that $\mathrm{W}_{\mathrm{k}-2, \mathrm{k}-1}=\mathrm{W}_{\mathrm{k}-1}=\mathrm{W}_{0, \mathrm{k}}, \mathrm{k}-=2,3, \ldots, \mathrm{n}$. In this case, $\mathrm{A}_{\mathrm{k}-1, \mathrm{k}}$ can be viewed as a relation $A_{k-1, k} \subset X_{k-1} \times X_{k} \times W$. For each pair $\left(x_{k-1}, X_{k}\right) \in X_{k-1} \times X_{k}, A_{k-1, k}\left(X_{k-1}\right.$, $\left.x_{k}\right)$ can be defined as the set $\left\{w \in W \mid\left(x_{k-1}, x_{k}, w\right) \in A_{k-1, k}\right\}$. Then $M_{k}$ and $\bigcirc_{k}$ can be well defined as in the above discussion. For a family of subsets $W_{s}$ and $\mathrm{W}_{\mathrm{s}, \mathrm{t}}(\mathrm{s} \in \mathrm{S}, \mathrm{t} \in \mathrm{T})$ of $\mathrm{W}$, if the following conditions

$$
\begin{aligned}
& \theta_{k}\left[\bigcup_{t \in T} \theta_{k-1}\left(\bigcup_{s \in S} W_{s, t}\right)\right]=\theta_{k-1}\left[\bigcup_{s \in S} \theta_{k}\left(\bigcup_{t \in T} W_{s, t}\right)\right], \\
& {\left[\theta_{k}\left(\bigcup_{s \in S} W_{s}\right)\right] \oplus_{k} W_{t}=\theta_{k}\left(\bigcup_{s \in S} W_{s} \oplus_{k} W_{t}\right),} \\
& W_{t} \oplus_{k-1} \theta_{k-1}\left(\bigcup_{s \in S} W_{s}\right)=\theta_{k+1}\left(\bigcup_{s \in S} W_{s} \oplus_{k-1} W_{t}\right)
\end{aligned}
$$

are satisfied, the optimum operators $\theta_{k}$ are called mutually commutative, $\oplus_{k}$ are called associative, $\theta_{k}$ and $\oplus_{k}$ are called distributive.

T heorem 7. If the optimum operators $\theta_{k}$ are commutative, $\oplus_{i}$ are associative, and they are distributive when combined together, then

$$
M_{k}=A_{0,1} \odot_{1} A_{1,2} \odot_{2} A_{2,3} \odot_{3} \cdots \odot_{k-1} A_{k-1, k} .
$$

Corollary. Under the conditions of Theorem 7, if $\theta=\theta_{k}$ and $\oplus=\oplus_{k-1, k^{\prime}}$ $\mathrm{k}=1,2, \ldots, \mathrm{n}$, then all $\odot$ 's are the same, and 
Kybernetes 26,3

$$
M_{k}=A_{0,1} \odot A_{1,2} \odot A_{2,3} \odot \cdots \odot A_{k-1, k}
$$

The other types of generalized dynamic programming models such as nondeterministic and continuous models can be similarly established with optimum operators and transformations.

\section{Remarks}

For most practical dynamic programming problems, if the criteria index sets are $\mathbf{R}$ or $\mathbf{Z}$, the optimum operators are usually minimization or maximization, and the criteria index transformation functions $F$ are usually additive, then the necessary and sufficient conditions are obviously satisfied. Therefore dynamic programming works effectively.

One of the advantages of the general dynamic programming is that it can deal with some multi-objective optimization problems. In this case, the criteria index set is usually an $\mathrm{n}$-dimensional vector space $\mathbf{R}^{\mathrm{n}}$, and the optimum operators on $\mathbf{R}^{\mathrm{n}}$ are Pareto minimization or maximization. A nother advantage of the general dynamic programming is that some selection problems can be treated like the example. The algorithm provided there actually has a polynomial time. In fact, if there are $k$ stages, and at each stage there are $n$ states, then the computational complexity can be figured out to be at most a polynomial of $n^{2} k^{2}-2 n^{2} k+n^{2}+n k$, that is, $O\left(n^{2} k^{2}\right)$. It is obvious that the complexity of dynamic programming is a polynomial time. The set-language treatment for dynamic programming in this paper may not be necessary in common practical use, but it clarifies some questions about the the foundation of dynamic programming. The Bellman principle of optimality cannot be accepted universally, though it is natural and philosophically subtle. We conjecture that an optimization problem of discrete type can be solved in a polynomial time if and only if it can be solved by the generalized dynamic programming with the construction of appropriate optimum operators.

\section{References}

Bellman, R. (1957), Dynamic Programming, Princeton University Press, Princeton, NJ.

Bellman, R. and Dreyfus, S. (1962), A pplied Dynamic Programming, Princeton University Press, Princeton, NJ.

Boltyanski, V.G. (1966), "Sufficient conditions for optimality and justification of dynamic programming method", SIAM Journal of Control, Vol. 4, pp. 326-61.

Chen, B. and Wu, S.Z. (1984), "On the problem of equilibrium under quasi-ordered preferences: applications of pansystems methodology to economics", Exploration of Nature, Vol. 7, pp. 29-36.

Denardo, E.V. (1967), "Contraction mappings in the theory underlying dynamic programming", SIAM Review, Vol. 9, pp. 165-77.

Furukawa, N. (1980), "Characterization of optimal policies in vector-valued Markovian decision process", M athematics and Operations Research, Vol. 5, pp. 271-9.

Henig, M.I. (1983), "Vector-valued dynamic programming", SIAM Journal of Control Optimization, Vol. 21, pp. 490-9. 
Hu, T.C. (1982), Combinatorial A lgorithms, A ddison-Wesley, Reading, M A .

Jackson, R. (1963), "Comments on the paper: Optimum cross-current extraction with product recycle, Letter to the Editor", Chemical Engineering Science, Vol. 18, pp. 215-7.

Qin, G.G. (1981a), "Some application of pan-graph and pansystem operation principle to M arkov process and optimization problems", Scientific Exploration, Vol. 3.

Qin, G.G. (1983), "Some applications of pansystems operation projection principle to dynamic games", Scientific Exploration, Vol. 4, pp. 59-64.

Qin, Y.Y. (1981b), "On the jar-metric principle (I), (II)", Science Exploration, Vol. 1, pp. 59-76, 101-8.

Rudd, D.F. and Blum, E.D. (1962), "Optimum cross-current extraction with product recycle", Chemical Engineering Science, Vol. 17, pp. 277-81.

Wu, C.P. (1980), "Multi-criteria dynamic programming", Scientia Sinica (A ), Vol. 23, pp. 338-95.

Wu, S.Z. and Wu, X.M . (1984), "Generalized principle of optimality in pansystems network analysis", Kybernetes, Vol. 13, pp. 231-5.

Wu, S.Z. and Wu, X.M. (1986), "Generalized fundamental equations in pansystems network analysis", Kybernetes, Vol. 15, pp. 181-4.

Wu, X.M . (1982), "Pansystems methodology: concepts, theorems and applications, (I)-(III)", Science Exploration, Vols. 1, 2, 4.

Wu, X.M. (1983), "Pansystems methodology: concepts, theorems and applications, (IV)-(V)", Science Exploration, Vols. 1, 4.

Wu, X.M . (1984a), "Pansystems methodology: concepts, theorems and applications, (VI)", Science Exploration, Vol. 1.

Wu, X.M. (1984b), "Pansystem metatheory of ecology, medicine and diagnostics (III)", Exploration of Nature, Vol. 1, pp. 13-20. 